\title{
Simulations of Electron Avalanches in an Ultra-Low-Background Proportional Counter
}

\author{
John W. Robinson ${ }^{\mathrm{a}}$, Craig Aalseth ${ }^{\mathrm{a}}$, Michael P. Dion ${ }^{\mathrm{a}, 1, *}$, Cory Overman ${ }^{\mathrm{a}}$, \\ Allen Seifert ${ }^{\mathrm{a}}$, Brent VanDevender ${ }^{\mathrm{a}}$ \\ ${ }^{a}$ Pacific Northwest National Laboratory Richland, WA 99354 USA
}

\begin{abstract}
New classes have been added to the simulation package Garfield ++ to import the potential and electric field solutions generated by ANSYS® Maxwell ${ }^{\mathrm{TM}}$ v.16. Using these tools we report results on the simulation of electron avalanches and induced signal waveforms in comparison to experimental data of the ultra-lowbackground gas proportional counters being developed at Pacific Northwest National Laboratory. Furthermore, an improved mesh search algorithm based on Delaunay triangulation was implemented and provided at least a three order of magnitude time savings when compared to the built-in point-location search class of Garfield++.
\end{abstract}

Keywords: gas proportional counter, ultra-low-background, detector modeling and simulation, Garfield, gaseous detectors

\section{Introduction}

There is ongoing research at Pacific Northwest National Laboratory (PNNL) to develop a new capability with ultra-low-background gas proportional counters (ULBPC). The detectors are constructed from ultra-pure electroformed copper that is grown in the 30 meter-water-equivalent underground laboratory at PNNL [1]. The electroforming process reduces the majority of uranium and thorium contamination in the copper to produce extremely low-background starting material. These counters are being developed for ground water dating with ${ }^{39} \mathrm{Ar}$ and age dating with ${ }^{14} \mathrm{C}$ and ${ }^{3} \mathrm{H}[2]$.

The applications described above demand an understanding of every pulse registered in the active gas volume to reduce the minimum detectable activity of the system. This includes electron avalanche response from a particle of interest interacting in the gas volume, background induced signals, micro-discharge pulses and other gas breakdown phenomena. In parallel to the experimental

\footnotetext{
${ }^{*}$ Corresponding author

Email address: michael.dion@pnnl.gov (Michael P. Dion)

${ }^{1}$ Address: P.O. Box 999 MSIN: J4-65 Tel.: +1-509-372-6983
} 
effort, simulations can also provide insight to geometrical construction and materials used to increase the fiducial volume while understanding spurious pulses (e.g., gas induced breakdown).

The electric field of a cylindrical, single-wire proportional counter can be solved analytically for much of its length (i.e., $E(r) \approx \frac{1}{r}$ ) but regions near the ends of the wire can present unforeseen behavior and dielectric material imposed on the geometry increase the difficulty in analytical methods. There are computational methods that can accurately solve (e.g., see Refs. [3, 4]) this (non-linear) field configuration with dielectric materials. Another more popular technique for a numerical solution of the electric potential and hence the electric field for a detector model is the finite element method (FEM). The FEM divides an object of interest into smaller elements, in this case tetrahedra, and then calculates the potential and electric field vectors at the nodes which can then be used to interpolate the electric field within each element. Increasing the number of tetrahedra increases the accuracy of the field solution, allowing areas of interest such as the anode wire of a gas proportional counter to be modeled in greater detail than other regions of a model. ANSYS® Maxwell ${ }^{\mathrm{TM}}$ v.16 [5] is available at PNNL for a variety of projects to perform electromagnetic modeling. An advantage of using Maxwell is the ability to import the full CAD model of the detector and subsequently solve the potential field. However, implementing FEM to solve the (non-linear) potential of a proportional counter with a linear interpolation can lead to singularities. A common method to "force" an accurate solution near the wire surface is the introduction of "dummy" cylinders into the model, as described in Section 3.1.

Multi-wire proportional chambers, time-projection chambers and single-wire proportional counters are used for measurements in many domains. Garfield [6] was developed to allow interfaces between finite element analysis solutions and a program providing Monte-Carlo techniques for electron-ion drift and subsequent signal development of a gas detector. Recently, Garfield has been transitioned to a $\mathrm{C}++$ based program known as Garfield ++ (termed here $\mathrm{G}++$ ) which is used for simulations in this work. $\mathrm{G}++$ has updated transport code (compared to the Fortran version) and a ROOT [7] interface. Magboltz [8] is called by $\mathrm{G}++$ and calculates the parameters of a user defined gas mixture (e.g., the electron drift velocity and Townsend coefficients as a function of electric field) and HEED [9] calculates the ionization created from a particle interaction in the detector medium (primary electron/ion pairs). The Monte-Carlo simulation of electron avalanches is computational intensive and time consuming, especially when the FEM mesh becomes finely spaced and contains many elements (orders of $10^{6}$ ). This is not an unknown or new problem and other works have implemented techniques to reduce computational time by orders of magnitude. For example, Bonzi et al. found several orders of magnitude (time) improvement by assuming an exponential distribution of electron path lengths between ionizing collisions [10]; eliminating the calculation between successive steps. Also, Alkaa et al. described a technique to reduce computation time by an order of magnitude by eliminating numerical integration of Newtons equations due to the cylindrical symmetry of proportional counters (at pressures above $0.1 \mathrm{~atm}$ ) [11]. 
We report the successful results of an effort that produced a simulation path from the computer-aided design (CAD), to accurate finite element method (FEM) solutions of the electric potential of the detector that were then imported into $\mathrm{G}++$. We have reduced the electron avalanche simulation time of $\mathrm{G}++$ by three orders of magnitude by implementing a new point-search algorithm based on a stochastic walk [12] and the Delaunay triangulation. Single-electron and avalanches induced by photon absorption were simulated in a mixture of $3 \mathrm{~atm}$ $90 \% \mathrm{Ar} 10 \% \mathrm{CH}_{4}$ (P-10). The gas gain extracted from the simulations is compared to experimental data using a Diethorn plot [13]. Furthermore, simulations provided induced signals in the detector which were then further processed to be directly compared to experimental waveforms acquired by the data acquisition system.

\section{The ULBPC Apparatus}

This section gives a brief description of the ULBPC, more details can be found in Ref. [2]. The detectors are manufactured from ultra-pure electroformed copper which is grown at PNNL [1]. The "crimp end" of the detector is shown in Figure 1. This end contains a dielectric spring structure (light blue structure in Figure 1) that centers a small copper cylinder which is crimped around the anode wire inside the cathode wall. The ULBPC gas gain was experimentally measured using $\approx 100 \mu \mathrm{Ci}$ of ${ }^{241} \mathrm{Am}$ in sealed source form. The ULBPC was filled with ultra-high purity $(99.997 \%)$ pre-mixed P-10 (uncertainty of $\pm 5 \%$ on the $\mathrm{CH}_{4}$ component) to 2286.38 torr $(3.01 \mathrm{~atm})$ [14]. At this pressure, the wire was biased from $+2150-2500 \mathrm{~V}$ in $50 \mathrm{~V}$ increments (counter wall held at ground) for gas gain measurements. Prior to final gas filling, the detector was evacuated to $\sim 0.1$ torr and backfilled to $3 \mathrm{~atm}$ with $\mathrm{P}-10$ three times. The ${ }^{241} \mathrm{Am}$ allows measurement of the $59.5 \mathrm{keV}$ gamma-ray and also K-shell fluorescence x-rays (8.05 keV [100\%], $8.03 \mathrm{keV}[\approx 51 \%]$ and $8.9 \mathrm{keV}[\approx 17 \%]$ ) [15] from the copper cathode wall which then interact in the gas volume. The ${ }^{241} \mathrm{Am}$ sealed source was placed directly against the cathode wall and midway along the wire length to reduce any end effects. A CANBERRA 2006 charge sensitive preamplifier was connected to an SHV-5 bulkhead connector which connects to the wire of the detector through a compression fitting [2]. The preamplifier has adjustable charge sensitivity of either 47 or $235 \mathrm{mV} /$ Mion-pair (47 for this work), diode protected field-effect transistor, pole-zero adjustment and low noise/fast rise time over a wide range of input capacitance making this a good choice for a proportional counter. Specific details of the preamplifier can be found in the user manual [16]. The output analog waveforms of the preamplifier were digitized with a $75 \mathrm{MHz}$ (1023 samples) Pixie-4 acquisition card [17]. The digital trapezoidal filter had an energy filter flat top of $4.68 \mu \mathrm{s}$, rise time of $20.85 \mu \mathrm{s}$ and a trigger filter flat top and rise time of $0.1064 \mu \mathrm{s}$. Waveforms of $13.6 \mu \mathrm{s}$ with a pre-trigger of $1.5 \mu$ s were digitized, analyzed and stored for every event above the trigger threshold. The Pixie-4 ADC channel-to-voltage calibration was accomplished in parallel to taking gas gain spectra and consisted of injecting a fixed charge into the preamplifier and measuring the Pixie-4 ADC channel number. This allowed 
each photopeak in the energy MCA to be converted from digital channel number to voltage. The experimental energy spectra of each applied voltage was analyzed using ROOT [7] by manually applying Gaussian fits to each peak of interest (8 and $59.5 \mathrm{keV}$ photopeak and test pulse). Subsequently, the number of secondary electrons created in the electron avalanche was calculated using the charge sensitivity of the preamplifier from which the gas gain was derived.

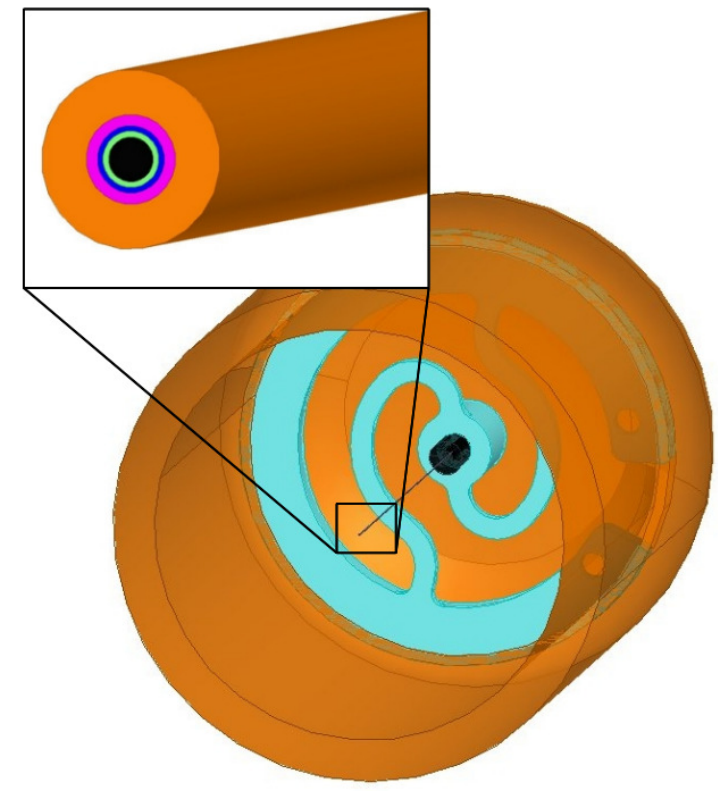

Figure 1: Cross section of the model used in simulations with a zoom on the dummy cylinders used to control the meshing process. The proportional counter wire was $25 \mu \mathrm{m}$ in diameter and the cathode had an inner diameter of $2.54 \mathrm{~cm}$. The center black cylinder is the anode wire and the green, blue, magenta, and orange cylinders represent imaginary surfaces of vacuum where Maxwell must form a 2D surface mesh.

\section{Prototyping method and Simulations}

\subsection{Finite Element Modeling in Maxwell}

To simulate the gas gain of the ULBPC, a geometrical model of the physical device was created in SolidWorks@. The SolidWorks model was exported in ACIS-SAT format then imported into Maxwell where materials and boundary conditions are assigned. Maxwell iteratively generates the finite element mesh using second-order tetrahedra until the solution reaches a set minimum error 
energy. To reduce simulation time and focus on areas of interest, the simulations presented in this work modeled about one-tenth of the ULBPC design as shown in Figure 1.

Preliminary models of the ULBPC solved with Maxwell were accurate in low-field regions; however, high-field regions near the anode wire were distorted and unsuitable for simulations. Field distortions were caused by tetrahedral elements with high aspect ratios, meaning the ratio of their longest and shortest dimensions is much greater than one. To reduce aspect ratios and improve accuracy, "dummy" structures were introduced to manually force the creation of a $2 \mathrm{D}$ mesh at these surfaces. As shown in Figure 1, the dummy structures in the detector model were concentric cylinders running along the wire. Strategic selection of the radius of each dummy cylinder and the number of line segments used to approximate curved objects in the model kept the aspect ratio near one for most elements in the final mesh. The radii of the cylinders were set to $1.25,1.5,2$ and 4 times the radius of the wire $(12.7 \mu \mathrm{m})$. The cylinders were approximated with line segments covering 15 degrees of the surface. This led to the tetrahedra of the mesh adjacent to the wire surface to have one edge of approximately one quarter of the wire radius. Thus setting the dummy cylinder with 1.25 times the wire radius led to an aspect ratio close to one. Radii of the outer dummy cylinders were set in similar way. The gas mixture was $3 \mathrm{~atm}$ P-10 for all simulations. Another parameter available in $\mathrm{G}++$ is the Penning ratio. The Penning effect is a mechanism where a metastable excited state of a gas atom (i.e., $\mathrm{Ar}^{*}$ ) can ionize the quench gas (i.e., methane) and therefore increase the number of secondary electron/ion pairs during the multiplication process. Without the presence of a quench gas this energy would be lost and therefore less electron/ion pairs would be generated. The Penning ratio was set at 0.24 , an approximation based on the work of Şahin et al. [18]. Simulations were performed at typical experimental operating parameters given in Section 2 .

\subsection{Data conversion and structure}

The Maxwell solution files containing the potential, electric field and boundary conditions must be imported into $\mathrm{G}++$ for subsequent electron and ion drift simulations. The Fortran version of Garfield stopped updating the model import code at Maxwell v.11 and $\mathrm{G}++$ also lacked a class for the v.16 import. Two paths were identified to interface Maxwell v.16 and $\mathrm{G}++$ : either a new class could be written to directly import the v.16 data into $\mathrm{G}++$ or the data could be converted to an intermediary format prior to import. The latter approach was chosen to eliminate redundancies in the original data, reducing load time in subsequent simulations. The general ASCII format of Gmsh [19], a 3D opensource finite element mesh program, was chosen as the intermediate format as it is well documented and allows viewing of the mesh outside of Maxwell and $\mathrm{G}++$ for verification. The Maxwell mesh converted into Gmsh format for the counter geometry is shown in Figure 2. Software engineers from ANSYS provided assistance by developing a Matlab script which sorted the mesh solution. The code was converted into Python to be run from the interpreter built into Maxwell. Enhancements were also made to the script to include the midpoints of 


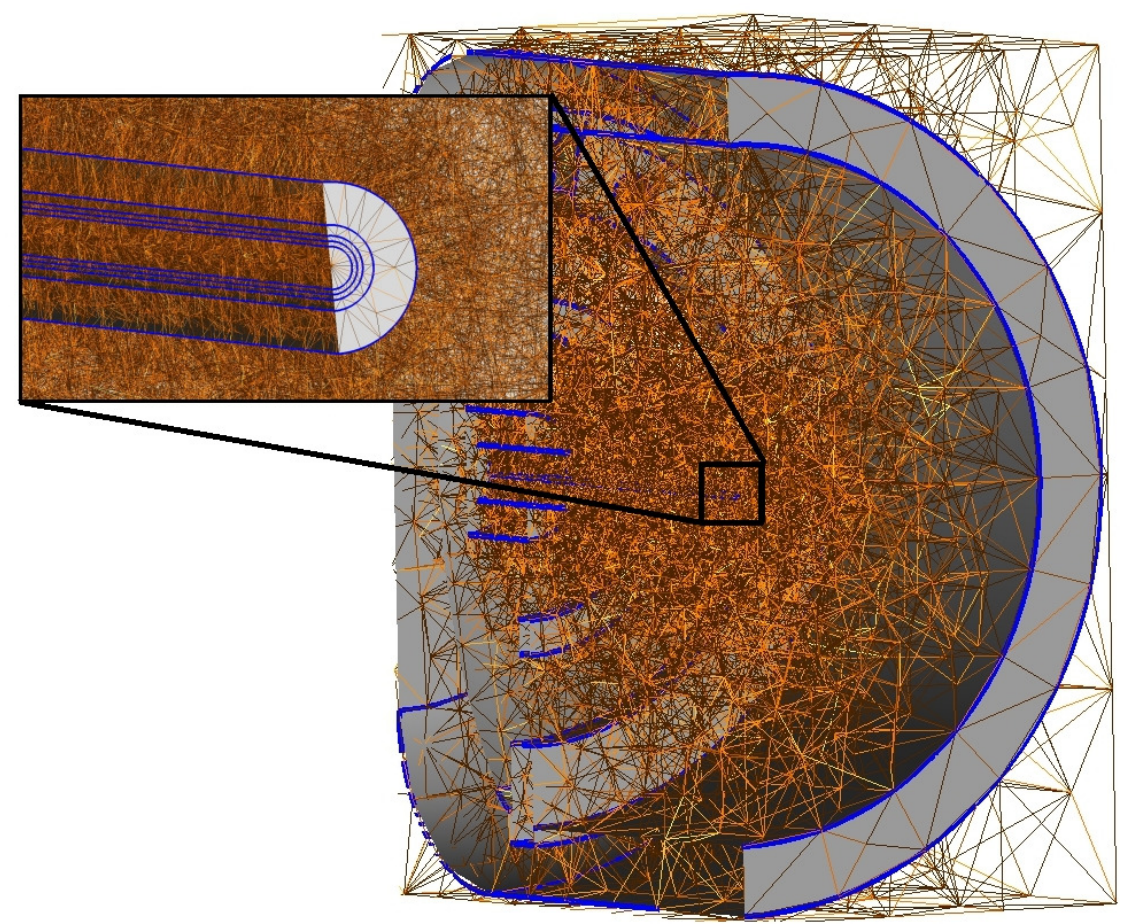

Figure 2: Gmsh rendering of the Maxwell mesh after importing into Gmsh. The dummy cylinders are visible in the zoomed portion of the figure and mesh boundary conditions are seen.

the second-order tetrahedra that could be used for more accurate interpolations in $\mathrm{G}++$. As noted by Veenhof [20], FEM programs have drawbacks associated with solving non-linear fields. In particular, the use of tetrahedral elements will most likely lead to a discontinuous electric field on tetrahedra boundaries. Incorporating the midpoints of the field solution suggests the interpolated field in $\mathrm{G}++$ is continuous across normal tetrahedral boundaries. A comparison of the magnitude of the electric field solved with Maxwell to the interpolation after import into $\mathrm{G}++$ is shown in Figure 3. The $\mathrm{G}++$ solution is shown as the solid (black) curve and the Maxwell solution is the dash (red) curve. Overall, there is good agreement between the two solutions except at the dummy cylinder boundaries (singularities).

\subsection{Mesh structures in Garfield ++}

The basic finite element field class in $\mathrm{G}++$, ComponentFieldMap, uses a simple unstructured mesh: an array for the mesh elements and an array for the element nodes. Existing FEM import classes with second-order tetrahedra available in $\mathrm{G}++$, especially the ComponentElmer class contributed by Renner [21] 


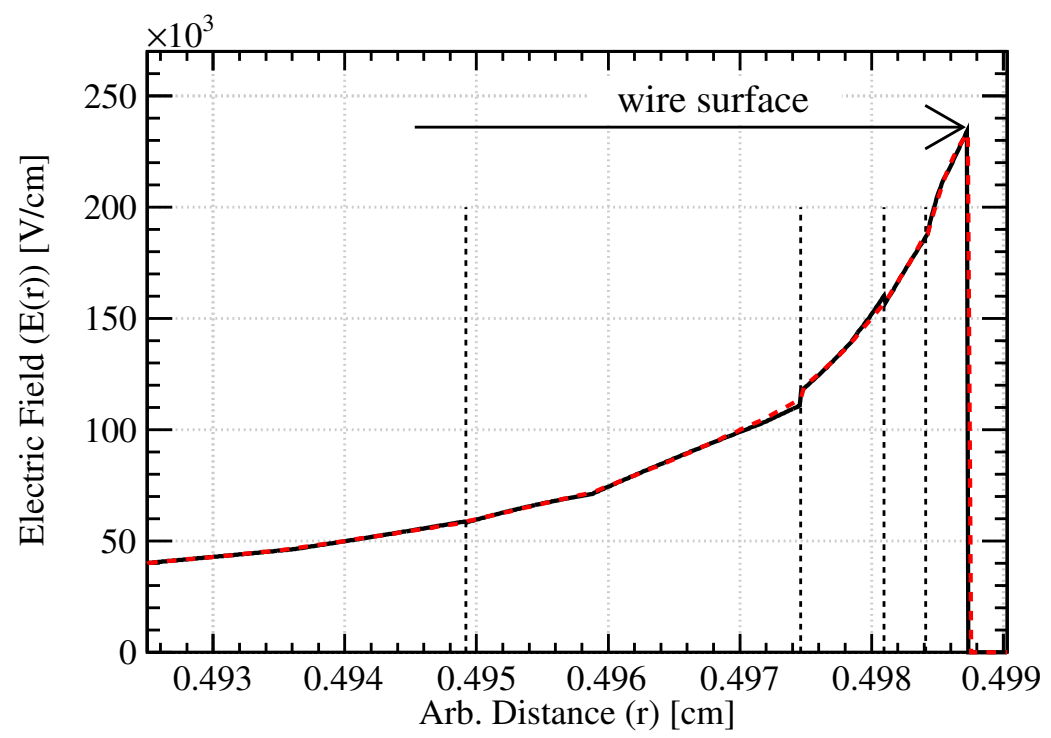

Figure 3: Plot of the magnitude of the electric field, $E(r)$, as a function of $r$; i.e., from the cathode wall to the wire surface. The $\mathrm{G}++$ interpolated solution is the solid (black) curve and the Maxwell solution is the dash (red) curve. The line plot was made at a coordinate absent from end effects. The dummy cylinder edges are indicated by the vertical dash lines.

and the ComponentAnsys123 class added by Schindler [22], provided the template for the new component class developed for the Gmsh files created from Maxwell models, i.e., ComponentGmsh.

Computation time for larger meshes is dominated by searching for the tetrahedron that contains a particular point, referred to as a point-location search. Models used in this work contained in excess of a million elements and it was apparent in early trials that the existing point-location algorithm needed modification to enable use of larger meshes. Since Maxwell uses straight-edged tetrahedra, ComponentGmsh was able to use the less computationally intensive search algorithm used by first-order tetrahedra. Additionally, the mesh was modified to store the minimum and maximum $\mathrm{X}, \mathrm{Y}$, and $\mathrm{Z}$ values of each tetrahedron reducing redundant calculations which further decreased computation time.

The afore mentioned modification decreased simulation time but the underlying search algorithm was still impractical for larger meshes. Thus a new class was written on the premise that Maxwell's meshes meet the requirements for Delaunay triangulation, specifically that the meshes are convex, i.e., the model cannot contain any internal holes or indentations in the outer boundary. Two new classes, DelaunayGmsh and its parent DelaunayFieldMap, were written using a structured mesh in which each element contained information about neighboring tetrahedra. The improved search algorithm works by stepping to 
neighbors that are progressively closer to the point of interest. The specific algorithm can be referred to as a "stochastic walk" as it randomizes orientation tests to prevent infinite search loops in non-Delaunay meshes [12]. To the authors knowledge this technique has not been applied to gaseous detectors before but Delaunay triangulation has been applied to solid-state detectors [23]. For a more detailed description of the Delaunay method see Ref. [24].

Maxwell does not use a true Delaunay triangulation though we found the results behaved similarly so a "visibility walk" similar to that described in Devillers et al. [12] worked for most cases. This work initially relied on the visibility walk and would fall back on a global search when infinite loops were encountered. Though the stochastic walk can have search times that scale exponentially with number of elements [12], Maxwell does not create the specialized configurations that cause exponential search length. The results of the global search algorithms and the stochastic walk are compared in Table 1. The authors expect other finite element packages will produce meshes that are similar as long as high aspect ratio elements are avoided.

Table 1: Speed improvements of drifting 1000 electrons using AvalancheMC in the proportional counter model starting $1.2 \mathrm{~cm}$ from the anode wire.

\begin{tabular}{|l|c|}
\hline Algorithm & Time for 1000 electrons $(\mathrm{s})$ \\
\hline ComponentGmsh, min/max calculated & 43600 \\
ComponentGmsh, min/max stored & 6200 \\
DelaunayGmsh & 21.0 \\
\hline
\end{tabular}

\section{Results and Comparison to Measurements}

\subsection{Avalanche Simulations and Gas Gain}

An electron avalanche is the underlying mechanism for signal generation in gas proportional counters. The absorption of a photon or the ionization from a charged particle creates primary electron/ion pairs in the gaseous medium. In $\mathrm{G}++$ the number of primary charge is calculated by HEED [9]. The charge drift under the influence of an applied electric field to the corresponding detector electrodes. The electrons are accelerated towards the wire and gain enough energy to ionize additional gas atoms (usually a noble gas based mixture) and begin an avalanche. The drift motion of the electrons and ions generated from the avalanche induce a current on the wire that is detected by electronics. The majority $(\approx 99 \%)$ of the signal induced in a traditional coaxial proportional counter is due to the relatively slow drift velocity of the ions and long drift path.

Avalanche simulations can be performed with either the AvalancheMC or AvalancheMicroscopic classes in $\mathrm{G}++$. In this work the AvalancheMC class was used for the drifting of ions and The AvalancheMicroscopic class was used for electron avalanche simulations since it is generally more accurate given its more rigorous treatment of collisions and ionization. 
One method of avalanche simulations was performed by drifting electrons from a discrete point in the active gas volume of the ULBPC model to the wire surface for subsequent avalanche formation. The number of electrons created in each avalanche was populated in a histogram that produces a single-electron spectrum (SES). The Polya distribution from the work of Jahoda et al. [25] was used to fit to the SES distribution:

$$
P(q, \mu, h)=\frac{1}{\mu} \frac{h^{h}}{\Gamma(h)} \cdot\left(\frac{q}{\mu}\right)^{h-1} \exp \left(\frac{-h q}{\mu}\right),
$$

where $q$ is the number of electrons produced in a single avalanche, $\mu$ is the mean number of electrons produced (the gas gain $M$ ), $h$ is a width or shape parameter, and $\Gamma$ is the gamma function. In Eq. (1), $h$ can also be related to the relative variance on the gain, $f$, as [26]:

$$
h=1 / f .
$$

The SES was based on $2.5 \times 10^{5}$ electron avalanches originating at a $1.2 \mathrm{~cm}$ radius from the anode wire (the cathode wall is a radius of $1.25 \mathrm{~cm}$ ) and $1.6 \mathrm{~cm}$ from the wire crimp along the wire axis to reduce end effects. It is assumed that this location is a good representation of the majority of the counter volume. The resulting SES distribution is shown in Figure 4, ignoring avalanches with zero gain. Net zero gain avalanches result from electron attachment, drift outside of the model sensitive volume or hitting the cathode wall. A Polya curve defined in Eq. (1) was fit to the SES distribution and the average gain of the detector was $1209 \pm 7$. In addition to the SES, the gas gain was also modeled by photon absorption using the HEED interface. To represent the different photon energies, HEED simulations were run with 1000 photons at $8.05 \mathrm{keV}, 500$ at $8.03 \mathrm{keV}$, and 170 at $8.9 \mathrm{keV}$. The method using HEED will generate a Gaussian distribution similar to what is measured experimentally. Figure 4 also contains a histogram of the simulated gas gain of full photon absorption and primary electron/ion pair calculations using HEED. The gas gain using the HEED calculation was determined by dividing the total number of secondary electrons produced in the avalanche simulation by the number of primary electrons produced by the photon absorption in the gas. The latter quantity is equal to the ratio of the photon energy $(8.05 \mathrm{keV})$ by the mean energy needed to create an electron/ion pair in P-10, W-value of $26 \mathrm{eV} /(\mathrm{e}-/$ ion pair) [27]. A Gaussian curve was fit to the histogram yielding a mean gas gain of $1172 \pm 2$. The lower energy peak at a gas gain of $\sim 680$ is the argon escape peak equal to full photon absorption minus the Ar k-edge, i.e., $4.7 \mathrm{keV}$. The gain calculated using HEED is $3 \%$ lower compared to the value returned using SES. However, only using 310 primary electrons (i.e., the expected number of primaries for an $8.05 \mathrm{keV}$ photon) and not accounting for the higher-energy fluorescent photons should shift the gas gain higher than that calculated using SES. Therefore, a simple explanation for the lower calculated gas gain of HEED comes from the number of primary electrons determined by HEED compared to the number expected by simple division of photon energy by 

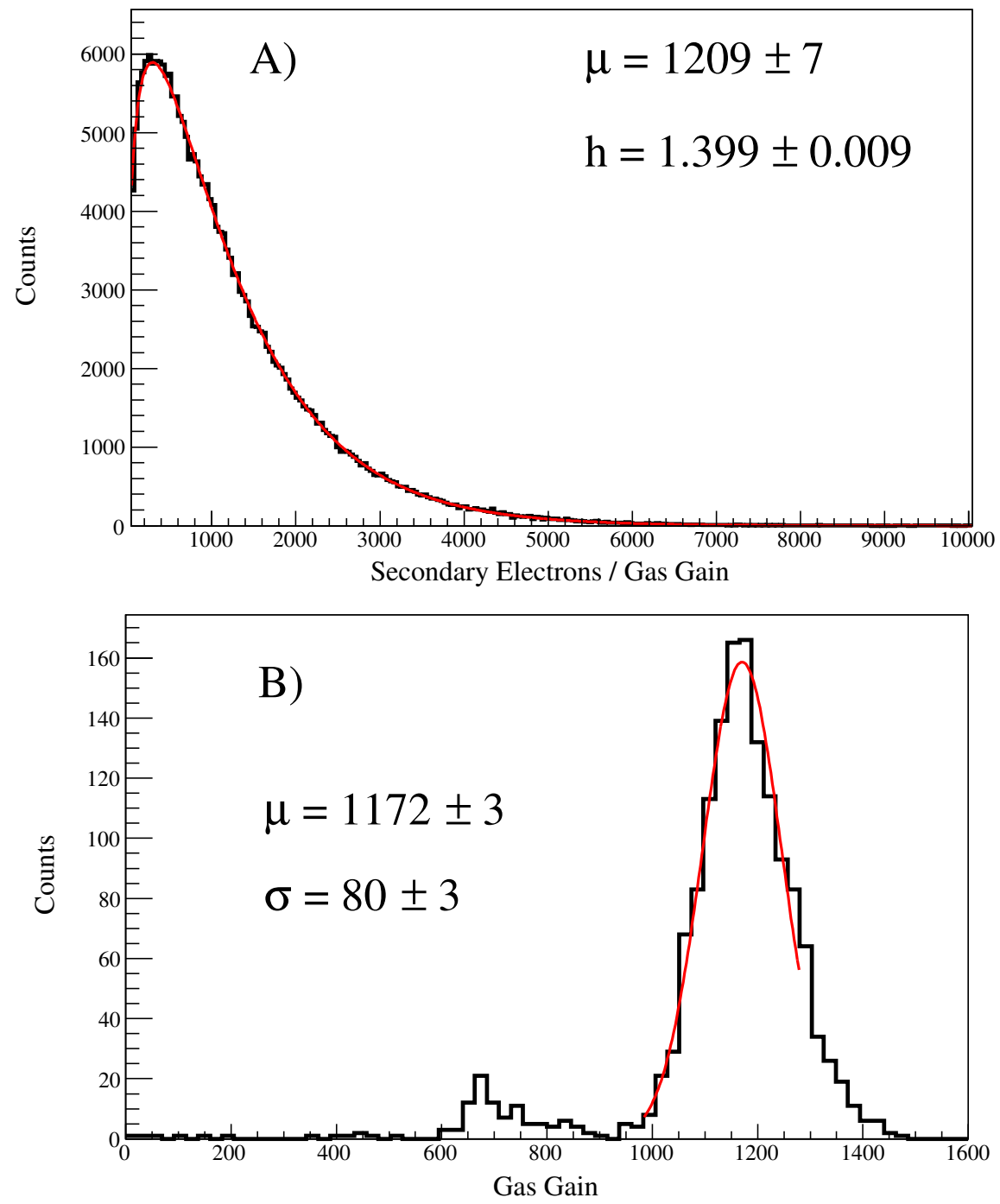

Figure 4: A) G++ SES used to extract the gain of the ULBPC model detector filled with $3 \mathrm{~atm}$ P-10 at an anode voltage of $+2150 \mathrm{~V}$. B) A distribution of the calculated gain for each avalanche originating from HEED photon events. The mean and standard deviation are found by a Gaussian fit to the data. 
the asymptotic $\mathrm{W}$-value. HEED often produces $\approx 3-4 \%$ fewer primary electrons, 299 for $8.05 \mathrm{keV}$ photons, which correlates to the $3 \%$ deficiency in difference amongst the two methods. Though the number of primary electrons produced by HEED could be used for simulated gain calculations, the value of 310 primary electrons was used to remain consistent with the experimental gain calculation of ULBPC.

The gas gain curves from simulations and experimental measurements from an ULBPC are shown as a Diethorn plot in Figure 5. The Diethorn formula is

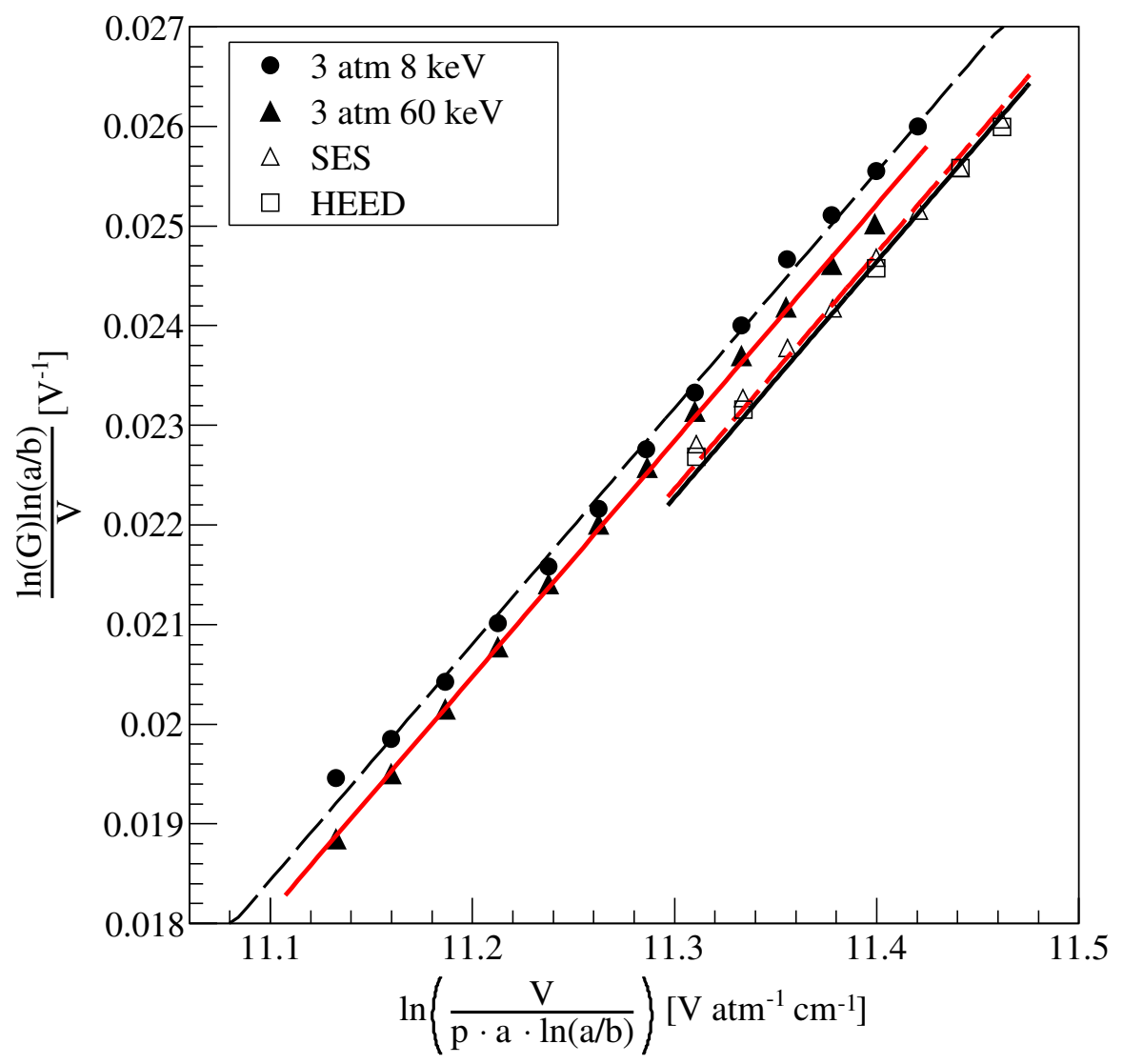

Figure 5: Diethorn plot for SES-based simulations, HEED-based simulations, and a proportional counter calibrated with copper $\mathrm{K}_{\alpha}$ lines $(8.05 \mathrm{keV}$ and $8.9 \mathrm{keV})$ or $59.5 \mathrm{keV}$ gamma-rays from ${ }^{241} \mathrm{Am}$.

defined as:

$$
\ln (G)=\frac{V}{\ln (b / a)} \cdot \frac{\ln (2)}{\Delta V}\left(\ln \frac{V}{p a \ln (b / a)}-\ln (K)\right),
$$


where $G$ is the measured gas gain, $a$ is the radius of the anode, $b$ is the radius of the cathode, $p$ is the gas pressure and $V$ is the applied voltage. The Diethorn parameters $K$ and $\Delta \mathrm{V}$ are the minimum electric field needed to start an electron avalanche and the potential difference an electron experiences between successive collisions, respectively [28]. There is good agreement between the Diethorn parameters of the experimental measurement and the simulation results as shown in Table 2 following linear fits to the data. If the gas gain at $+2150 \mathrm{~V}$ is compared between the mathematical average of gas gain from simulation and measurement, the simulated gain is $\approx 15 \%$ deficient. The difference in the gas gain for specific voltages between the simulations and measurements can most likely be attributed to the choice of (a constant) Penning transfer ratio in the simulation, uncertainty of the gas fill composition and/or pressure and other fine mechanical details of the actual detector that are not captured in the simulation (e.g. surface of the fine anode wire). Previous simulation studies have used multiple Penning ratios to calculate the value that best matches experiment [29] and the same method will likely be applied in future simulations of ULBPCs.

The $59.5 \mathrm{keV}$ data points at high gas gain diverge slightly from a linear relationship and this could be space charge effects. It has been shown that the relative channel shift due to space charge effects can be calculated for cylindrical proportional counters [30] and there is a dependence on pulse rate and photon energy (i.e., the number of primary electron-ion pairs created). The count rate during experimental testing was low $(\approx 1 \mathrm{~Hz})$ and therefore pile-up is not an issue. Detailed calculations for the ULBPC are planned in the future to determine if the photon energy is contributing to the observed disagreement.

Table 2: Diethorn fit parameters comparing the simulated and experimental measurements of the ULBPC in Figure 5 and the gas gain for all methods at $+2150 \mathrm{~V}$. The uncertainty on the Diethorn parameters only includes statistical standard deviation from the linear fit to the data. Experimental uncertainty is estimated at $\approx 15 \%$.

\begin{tabular}{|l|c|c|c|}
\hline Data set & $\left.K\left[\mathrm{kV} \mathrm{atm}{ }^{-1} \mathrm{~cm}^{-1}\right)\right]$ & $\Delta V[\mathrm{~V}]$ & Gain $+2150 \mathrm{~V}$ \\
\hline SES simulation & $31.4(1.1)$ & $29.3(0.8)$ & 1212.3 \\
HEED simulation & $31.5(1.2)$ & $29.3(0.8)$ & 1172.0 \\
Copper $K_{\alpha}$ & $30.4(0.4)$ & $29.2(0.3)$ & 1408.8 \\
${ }^{241}$ Am $59.5 \mathrm{keV}$ & $30.8(0.3)$ & $29.3(0.2)$ & 1327.3 \\
\hline
\end{tabular}

\subsection{Induced current and signal development}

The induced signal from the drifting of the liberated charge was simulated in two parts: the electron drift during avalanche and subsequent drifting of ions. G++ uses the Shockley-Ramo theorem to calculate the induced current produced by the movement of charged particles through the detector [31, 32]. The required weighting field is calculated in Maxwell by setting the potential of the anode wire to unity and ground potential elsewhere. The simulated induced current signal was further processed by a circuit simulator to mimic the front-end electronics of the detector (i.e., CANBERRA 2006 preamplifier). Therefore, the 

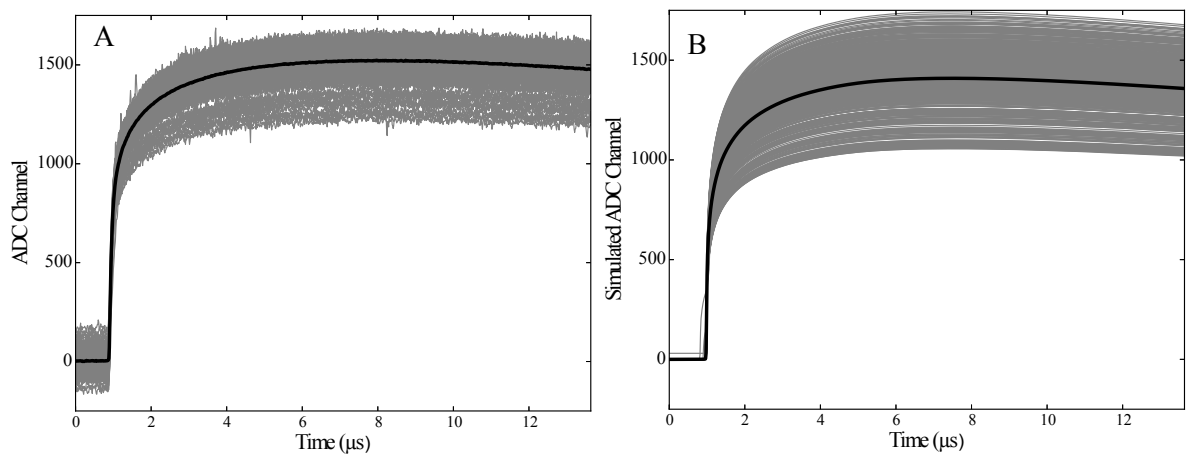

Figure 6: A: Plot of multiple signals collected from a ULBPC using 3 atm P-10. The black line shows the mean signal based on a point-by-point Gaussian fit of the signals. B: Simulated signals using the same conditions.

induced current on the anode wire was simulated using an avalanche of multiple primary electrons produced by photoionization in HEED. A single photon with energy $8.05 \mathrm{keV}$ was released into the detector, producing primary electrons that were subsequently avalanched microscopically; ions produced by the avalanche were then drifted toward the cathode wall using the Monte-Carlo method with time steps of $10 \mathrm{~ns}$ for a total of $14 \mu \mathrm{s}$. This provided an induced signal of sufficient time to compare to experimental waveforms acquired by the ULBPC data acquisition cards $(13.6 \mu \mathrm{s})$. Though ions continue drifting for $4-5 \mathrm{~ms}$, most of the induced signal develops in less than $100 \mu$ s making it acceptable to ignore most of the drift time. As a first approximation, the ion mobility for P-10 was set at $1.9 \mathrm{~m}^{2} \mathrm{~V}^{-1} \mathrm{~s}^{-1}$, which corresponds to the low-field limit given in Ref. [33].

The induced current signals that corresponded to full photon absorption (i.e., escape peak events were not considered) were used for further processing. The simulated signal is a sum of the electron and ion induced currents. The functional schematic of the preamplifier was modeled using Qucs, an open-source circuit simulator [34], and the output was compared to the experimental results. The simulation produced a table of time and induced current values. This data table was processed by Qucs that then produced a simulated output preamplifier signal (i.e., voltage) as a function of time. For ease of viewing and comparison,

the experimental induced signals from the ULBPC and the simulated waveforms were normalized to a baseline of zero using the average of their respective pretrigger baseline. The pulse rise time and amplitude of signals produced via simulations show similar characteristics to the experimental measurements. The mean amplitude of 1000 experimental waveforms was $1560 \pm 109$ ADC channels for the copper $\mathrm{K}_{\alpha}$ peak and 250 simulated pulses from $8.05 \mathrm{keV}$ photons had a mean amplitude of $1502 \pm 87$ ADC channels. Therefore, the mean amplitudes of the induced waveforms agree within uncertainty and also show the same (lower) trend of simulated gain compared to experimental. 


\section{Conclusions}

A simulation procedure was successfully developed to model the ULBPC manufactured at PNNL. First, a CAD model was imported into ANSYS Maxwell (version 16) to calculate the electric potential map. This solution was then imported into $\mathrm{G}++$ to simulate transport and avalanche phenomena in the gas volume. In order to accomplish this work, new classes were added to $\mathrm{G}++$ to properly import the Gmsh-formatted files. An entirely new point-location search algorithm was introduced involving a "stochastic walk" that requires far fewer steps to locate tetrahedra of interest. This new method lead to a large reduction in simulation time on the order of three orders of magnitude compared to the original algorithm.

The gas gain of the ULBPC was found to be $\approx 1200$ through simulations at a voltage of $+2150 \mathrm{~V}$ and $3 \mathrm{~atm} \mathrm{P}-10$ with a Penning ratio of 0.24 . The deviations between the simulated and experimental gas gain are likely due to the use of a fixed Penning ratio in the simulation, uncertainty in the first Townsend coefficient and the mechanical model used in the simulation not encompassing all fine mechanical details of the experimental apparatus (the anode wire surface) are responsible. The experimental and simulated gas gain was compared using a Diethorn plot. Within the statistical uncertainty, the Diethorn parameters are in agreement but there is a clear systematic offset between the experimental data $(8 \mathrm{keV}$ and $59.5 \mathrm{keV})$ and more significantly, between the simulated and experimental measurements. The offset comparing the experimental and simulated values could be due to the constant Penning ratio used in the simulation, or the use of a constant $\mathrm{W}$-value for the two photon energies (or a combination of these factors). The disagreement between the two experimental data sets can be explained by an electric field screening due to space charge effects of the $59.5 \mathrm{keV}$ data. Future work is expected to address items that can allow the simulations to correlate with experimental results of ULBPCs. Furthermore, the signal output of the preamplifier coupled to an ULBPC was modeled using the induced current from $\mathrm{G}++$ and the Qucs program. The gas gain of the signals generated by Qucs was calculated by sampling the amplitude (largest bin) and back-calculating the number of secondary electrons. This distribution exhibited the same (lower) trend that the microscopic avalanche simulations suggest in comparison to the experimental gas gain data.

Utilizing $\mathrm{G}++$ for the ULBPCs is a powerful toolkit for the future development of the low-background program at PNNL. This capability is envisioned as being able to predict detector response to geometrical changes in the detector volume eliminating costly trial and error experimental implementation. Examples include mechanical changes to the dielectric components, varying the anode wire diameter and changing the detector volume to support all applications covered by the ULBPCs. Future work includes adding random noise to the simulated waveforms at a level represented by the experimental apparatus, and analyzing the $\mathrm{G}++$ induced waveforms with the current ULBPC software to fine-tune the simulation parameters. Following the inclusion of (electrical) noise to the Qucs waveforms, the energy resolution of each simulated induced signal 
could be measured and compared to the experimental energy resolution. Efforts are currently underway to estimate the fiducial volume of the full proportional counter using the tools described in this work.

\section{Acknowledgments}

We would like to thank the application engineer Julius Saitz at ANSYS for enabling these simulations by helping us interpret the output data files from Maxwell v.16.

The research described in this paper is part of the Ultra-Sensitive Nuclear Measurements Initiative at Pacific Northwest National Laboratory. It was conducted under the Laboratory Directed Research and Development Program at PNNL, a multiprogram national laboratory operated by Battelle for the U.S. Department of Energy.

\section{References}

[1] E. Hoppe, C. Aalseth, O. Farmer, T. Hossbach, M. Liezers, H. Miley, N. Overman, J. Reeves, Nuclear Instruments and Methods in Physics Research Section A: Accelerators, Spectrometers, Detectors and Associated Equipment 764 (2014) 116 - 121. doi:10.1016/j.nima.2014.06.082.

[2] C. Aalseth, A. Day, E. Hoppe, T. Hossbach, B. Hyronimus, M. Keillor, K. Litke, E. Mintzer, A. Seifert, G. Warren, Journal of Radioanalytical and Nuclear Chemistry 282 (1) (2009) 233-237. doi:10.1007/s10967-009-0258-5.

[3] N. Majumdar, S. Mukhopadhyay, Nuclear Instruments and Methods in Physics Research Section A: Accelerators, Spectrometers, Detectors and Associated Equipment 566 (2) (2006) 489 - 494. doi:10.1016/j.nima.2006.06.035.

[4] S. Mukhopadhyay, N. Majumdar, Nuclear Science, IEEE Transactions on 53 (2) (2006) 539-543. doi:10.1109/TNS. 2006.872634.

[5] ANSYS@ Maxwell ${ }^{\mathrm{TM}}$, Release 16.0, Help System, ANSYS, Inc., http://www.ansys.com.

[6] R. Veenhof, Nuclear Instruments and Methods in Physics Research Section A: Accelerators, Spectrometers, Detectors and Associated Equipment 419 (23) (1998) 726 - 730. doi:10.1016/S0168-9002(98)00851-1.

[7] R. Brun, F. Rademakers, Proceedings AIHENP'96 Workshop, Lausanne, Sep. 1996, Nucl. Instr. and Meth. A 389 (1997) 81-86, see also http://root.cern.ch/.

[8] S. F. Biagi, MAGBOLTZ program, http://garfield.web.cern .ch/garfield/. 
[9] I. Smirnov, Nuclear Instruments and Methods in Physics Research Section A: Accelerators, Spectrometers, Detectors and Associated Equipment 554 (13) (2005) 474 - 493. doi:10.1016/j.nima.2005.08.064.

[10] E. V. Bonzi, R. Mainardi, J. Phys. D: Appl. Phys. 32 (4) (1999) 440-444. doi:10.1088/0022-3727/32/4/014.

[11] A. Alkaa, K. Mitev, P. Ségur, Nuclear Instruments and Methods in Physics Research Section A: Accelerators, Spectrometers, Detectors and Associated Equipment 580 (1) (2007) 161 - 164, proceedings of the 10 th International Symposium on Radiation Physics \{ISRP\} 10. doi:10.1016/j.nima.2007.05.074.

[12] O. Devillers, S. Pion, M. Teillaud, International Journal of Foundations of Computer Science 13 (02) (2002) 181-199. doi:10.1142/S0129054102001047.

[13] W. Diethorn, A Methane Proportional Counter System For Natural Radiocarbon Measurements, Master's thesis, Carnegie Inst. of Tech., Pittsburgh (Mar. 1956).

[14] Mesurement specialties, MSP-300-250. URL http://www.meas-spec.com/product/t_product.aspx?id=2888\#

[15] A. C. Thompson (Ed.), X-Ray Data Booklet, Lawrence Berkeley National Laboratory, University of California. URL http://xdb.lbl.gov/

[16] CANBERRA Model 2006 Proportional Counter Preamplifier. URL http://www.canberra.com/products/radiochemistry_lab/pdf/ Model-2006-SS-CSP0158.pdf

[17] XIA LLC, http:xia.com, 31057 Genstar Rd. Hayward CA 94544.

[18] Ö. Şahin, I. Tapan, E. Özmutlu, R. Veenhof, Journal of Instrumentation 5 (05) (2010) P05002. doi:10.1088/1748-0221/5/05/P05002.

[19] C. Geuzaine, J.-F. Remacle, International Journal for Numerical Methods in Engineering 79 (11) (2009) 1309-1331. doi:10.1002/nme.2579.

[20] R. Veenhof, Journal of Instrumentation 4 (12) (2009) P12017. doi:doi:10.1088/1748-0221/4/12/P12017.

[21] J. Renner, Detector simulation in Garfield++ with open-source finite element electrostatics (2012). URL http://garfieldpp.web.cern.ch/garfieldpp/examples/elmer/ garfield_elmer_doc.pdf 
[22] H. Schindler, C. Fabjan, Microscopic simulation of particle detectors, Ph.D. thesis, Vienna University of Technology (2012). URL https://cds.cern.ch/record/1500583/files/ CERN-THESIS-2012-208.pdf

[23] R. Agnese, D. Brandt, M. Asai, B. Cabrera, S. Leman, K. McCarthy, P. Redl, T. Saab, D. Wright, Journal of Low Temperature Physics 176 (5-6) (2014) 930-936. doi:10.1007/s10909-014-1182-9.

[24] M. de Berg, O. Cheong, M. van Kreveld, M. Overmars, Computational geometry: algorithms andapplications, Springer-Verlag, Berlin. doi:10.1007/978-3-540-77974-2.

[25] K. Jahoda, D. McCammon, Nuclear Instruments and Methods in Physics Research Section A: Accelerators, Spectrometers, Detectors and Associated Equipment 272 (3) (1988) 800-813. doi:10.1016/0168-9002(88)90762-0.

[26] M. Kobayashi, NIM A $562 \quad$ (1) (2006) $136 \quad-\quad 140$. http://dx.doi.org/http://dx.doi.org/10.1016/j.nima.2006.03.001 doi:http://dx.doi.org/10.1016/j.nima.2006.03.001.

[27] R. S. Wolff, Nuclear Instruments and Methods 115 (2) (1974) 461 - 463. doi: 10.1016/0029-554X(74)90247-X.

[28] W. Blum, L. Rolandi, Particle Detection With Drift Chambers, SpringerVerlag Telos, New York, NY, 1994.

[29] A. Takada, T. Tanimori, H. Kubo, J. Parker, T. Mizumoto, Y. Mizumura, S. Iwaki, T. Sawano, K. Nakamura, K. Taniue, et al., Journal of Instrumentation 8 (10) (2013) C10023. doi:10.1088/1748-0221/8/10/C10023.

[30] R. Hendricks, Rev. Sci. Instr. 40 (9) (1969) 1216-1223. doi:10.1063/1.1684203.

[31] W. Shockley, Journal of Applied Physics 9 (1938) 635-636.

[32] S. Ramo, Proc. Ire 27 (9) (1939) 584.

[33] G. Schultz, G. Charpak, F. Sauli, Revue de Physique Appliquée 12 (1) (1977) 67-70. doi:10.1051/rphysap:0197700120106700.

[34] M. Brinson, S. Jahn, International Journal of Numerical Modelling: Electronic Networks, Devices and Fields 22 (4) (2009) 297-319. doi:10.1002/jnm.702. 